\title{
Escurecimento da polpa em pera 'Rocha' influenciado pela composição mineral do fruto e condições de atmosfera controlada
}

\section{Flesh browning in 'Rocha' pear as affected by fruit mineral composition and controlled atmosphere conditions}

\author{
Mariuccia Schlichting De Martin ${ }^{1}$, Cristiano André Steffens ${ }^{2 *}$, Cassandro Vidal Talamini do \\ Amarante $^{2}$, Marília Farias Rodrigues ${ }^{2}$, Angélica Schmitz Heinzen ${ }^{2}$, Auri Brackmann ${ }^{3}$ \\ 1. Empresa de Pesquisa Agropecuária e Extensão Rural de Santa Catarina - Fisiologia e Tecnologia Pós-Colheita - Caçador (SC), Brazil. \\ 2. Universidade do Estado de Santa Catarina - Agronomia - Lages (SC), Brazil. \\ 3. Universidade Federal de Santa Maria - Fitotecnia - Santa Maria (RS), Brazil.
}

RESUMO: O objetivo deste trabalho foi avaliar o efeito de condições de armazenamento em atmosfera controlada (AC) e da composição mineral do fruto sobre ocorrência de escurecimento da polpa em pera 'Rocha'. Os frutos foram armazenados sob diferentes condições de $\mathrm{AC}: 0,5 \mathrm{kPa} \mathrm{O}_{2}+$ $<0,03 \mathrm{kPa} \mathrm{CO} ; 1,0 \mathrm{kPa} \mathrm{O}+<0,03 \mathrm{kPa} \mathrm{CO}_{2} ; 1,0 \mathrm{kPa} \mathrm{O}+1,0 \mathrm{kPa} \mathrm{CO}$; $1,0 \mathrm{kPaO}_{2}+2,0 \mathrm{kPaCO}_{2}$ e1,0 $\mathrm{KPaO}_{2}+3,0 \mathrm{kPaCO}_{2}$. Osfrutos foram avaliados quanto à incidência e severidade de escurecimento da polpa após 9 meses de armazenamento $\left(-0,5 \pm 0,1^{\circ} \mathrm{C}\right.$ e UR de $\left.96 \pm 2 \%\right)$. Posteriormente, frutos com e sem a presença do distúrbio foram avaliados quanto às concentrações de $\mathrm{Ca}, \mathrm{Mg}, \mathrm{K}, \mathrm{N}$ e as relações $\mathrm{K} / \mathrm{Ca}$, Mg/Ca e N/Ca. O armazenamento sob $1,0 \mathrm{KPaO}_{2}+3,0 \mathrm{kPaCO}$ proporcionou aos frutos maior incidência de escurecimento da polpa em relação aos armazenados sob $0,5 \mathrm{kPaO}_{2}+<0,03 \mathrm{kPaCO}_{2}$ e $1,0 \mathrm{kPa} \mathrm{O}_{2}+<0,03 \mathrm{kPa} \mathrm{CO}_{2}$, ocasionando ainda maior severidade do distúrbio em comparação às demais condições de armazenamento. Peras com escurecimento da polpa apresentaram menores concentrações deCa, bem como concentrações mais elevadas de Kemaiores relações dos elementos K/Ca,Mg/CaeN/Ca, comparativamente a frutos sem incidência do dano. Todavia, a análise multivariada de todos esses elementos minerais mostrou que a relação K/Ca foi a mais indicada para discriminar frutos sem e com escurecimento da polpa em peras 'Rocha'.

Palavras-chave: Pyrus communis, análise canônica discriminante, armazenamento, deficiência de cálcio, distúrbio fisiológico.
ABSTRACT: The aim of this study was to evaluate the effect of controlled atmosphere (CA) conditions and mineral contents on flesh browning in 'Rocha' pear. The fruits were stored at different CA conditions: $0.5 \mathrm{KPaO}_{2}$ $+<0.03 \mathrm{kPaCO}_{2} ; 1.0 \mathrm{kPaO}_{2}+<0.03 \mathrm{kPa} \mathrm{CO}_{2} ; 1.0 \mathrm{kPaO}_{2}+1.0 \mathrm{kPa} \mathrm{CO}_{2}$; $1.0 \mathrm{kPaO}_{2}+2.0 \mathrm{kPaCO}_{2}$ and $1.0 \mathrm{kPaO}_{2}+3.0 \mathrm{kPaCO}_{2}$. Fruit were evaluated for incidence and severity of flesh browning after 9 months of storage $\left(-0.5 \pm 0.1^{\circ} \mathrm{C}\right.$ and $\left.96 \pm 2 \% \mathrm{RH}\right)$. Also, fruit with and without the disorder were assessed for contents of $\mathrm{Ca}, \mathrm{Mg}, \mathrm{K}$, and $\mathrm{N}$, as well as the $\mathrm{K} / \mathrm{Ca}, \mathrm{Mg} / \mathrm{Ca}$, and $\mathrm{N} / \mathrm{Ca}$ ratios. Fruit stored at $1.0 \mathrm{kPa} \mathrm{O}_{2}+$ $3.0 \mathrm{KPaCO}_{2}$ had higher incidence of flesh browning than those stored at $0.5 \mathrm{kPaO}_{2}+<0.03 \mathrm{kPa} \mathrm{CO}$ and $1.0 \mathrm{kPa} \mathrm{O}+<0.03 \mathrm{kPa} \mathrm{CO}_{2}$, as well as higher severity of disorder than all the other storage conditions. Pears with flesh browning had lower Ca content, as well as higher $\mathrm{K}$ content and higher $\mathrm{K} / \mathrm{Ca}, \mathrm{Mg} / \mathrm{Ca}$, and $\mathrm{N} / \mathrm{Ca}$ ratios than fruit without the disorder. However, the multivariate analysis of all these mineral elements showed that the $\mathrm{K} / \mathrm{Ca}$ ratio provides the best discrimination between fruit with and without flesh browning in 'Rocha' pears.

Key words: Pyrus communis, canonical discriminant analysis, storage, calcium deficiency, physiological disorder. 


\section{INTRODUÇÃO}

O armazenamento em atmosfera controlada (AC) traz inúmeros benefícios para a conservação da pera 'Rocha', uma vez que a redução das pressões parciais de $\mathrm{O}_{2}$ e aumento das pressões parciais de $\mathrm{CO}_{2}$ retardam a senescência e mantêm a qualidade dos frutos por períodos mais prolongados (Martin et al. 2015). Contudo, o armazenamento sob condições com baixo $\mathrm{O}_{2}(<2,0 \mathrm{kPa})$ e alto $\mathrm{CO}_{2}(>0,7 \mathrm{kPa})$ pode favorecer a ocorrência de escurecimento da polpa nos frutos (Galvis-Sánchez et al. 2004), levando a consideráveis perdas econômicas.

O escurecimento da polpa em peras é caracterizado pela presença de manchas marrons, as quais podem tanto atingir pequenas porções da polpa quanto afetá-la de forma generalizada (Franck et al. 2007), podendo ocorrer a formação de cavidades na polpa (Lammertyn et al. 2003). Normalmente, os sintomas são notados apenas no momento do consumo, quando os frutos são cortados, mas, em casos mais severos, é possível visualizar externamente a ocorrência do distúrbio (Yan et al. 2013).

Acredita-se que o estresse, causado por condições inadequadas durante o armazenamento em AC, como pressões parciais muito reduzidas de $\mathrm{O}_{2}$ e/ou muito elevadas de $\mathrm{CO}_{2}$, induza a respiração anaeróbica nos tecidos da polpa, aumentando o conteúdo de etanol e acetaldeído, levando à formação de radicais livres, afetando o metabolismo energético e, consequentemente, danificando as membranas das células da polpa (Saquet e Streif 2006; Franck et al. 2007; Neuwald et al. 2008). Dessa forma, as causas do escurecimento da polpa em condições inadequadas de AC possivelmente estão associadas aos processos que afetam a integridade de membranas.

Alguns trabalhos associam a deficiência de Ca com a ocorrência de distúrbios fisiológicos em maçãs (Corrêa et al. 2012; Amarante et al. 2013), pois os mesmos exercem um importante papel na permeabilidade seletiva, na estruturação e na funcionalidade das membranas celulares, por meio da ligação de fosfolipídeos e de monogalactosídeos na superfície da membrana (Freitas et al. 2010; Miqueloto et al. 2011). As concentrações de Mg podem ter relação com o escurecimento da polpa, uma vez que esse elemento compete diretamente com o Ca nos sítios de ligação na membrana plasmática, apesar de não apresentar o mesmo papel fisiológico na manutenção da funcionalidade e estrutura das membranas (Freitas et al. 2010; Amarante et al. 2013). Conteúdos elevados de K (Neuwald et al. 2008; James e Jobling 2009) e de N (Amarante et al. 2010) também têm sido correlacionados com a presença de distúrbios fisiológicos em maçãs.

Não somente os elementos individualmente, mas as relações entre as concentrações de nutrientes na polpa também podem influenciar a predisposição dos frutos ao desenvolvimento de distúrbios fisiológicos (Amarante et al. 2006). Corrêa et al. (2012) observaram que maçãs 'Fuji' com maior relação $\mathrm{K} / \mathrm{Ca}$ apresentam maior predisposição ao escurecimento da polpa. Para maçãs 'Fuji' e 'Catarina', maiores valores das relações K/Ca e N/Ca foram observados na polpa de frutos que apresentaram incidência de bitter pit, em relação àqueles que não apresentaram o distúrbio (Miqueloto et al. 2011).

Poucos são os estudos que relacionam as concentrações minerais da polpa de peras com a incidência de distúrbios fisiológicos, porém alguns autores verificaram o efeito benéfico da aplicação pré-colheita de $\mathrm{CaCl}_{2}$ na sua prevenção (Mahajan e Dhatt 2004; Raese e Drake 2006). Em peras 'Conference', Wójcik (2012) observou que 6 aplicações précolheita de $\mathrm{CaCl}_{2}$ proporcionaram frutos menos maduros e menos sensíveis ao escurecimento da polpa em relação àqueles que não foram submetidos ao tratamento.

Este trabalho teve como objetivo avaliar o efeito de condições de armazenamento AC e da composição mineral do fruto sobre ocorrência de escurecimento da polpa em pera 'Rocha'.

\section{MATERIAL E MÉTODOS}

Os frutos foram colhidos em um pomar comercial localizado no município de Vacaria, RS (lat 28 30'39"S, long 50 55'47"W e $960 \mathrm{~m}$ de altitude), na safra 2012/2013. Foram utilizadas pereiras da cultivar 'Rocha', com 6 anos de idade, sobre porta-enxerto Marmelo 'BA29' (Cydonia oblonga) e espaçadas em 1,5 m na linha e 4,0 m entre linhas. Após a colheita, foram eliminados os frutos que apresentavam podridões, lesões, defeitos ou eram de baixo calibre.

No momento da colheita comercial, efetuou-se uma análise inicial de 3 amostras de 15 frutos em cada amostra, para determinação do estádio de maturação, através das análises de cor da casca, em 2 pontos opostos na região equatorial do fruto, firmeza de polpa, acidez titulável (AT) e teor de sólidos solúveis (SS). A cor da casca foi avaliada em termos de valores de ângulo 'hue' $\left(\mathrm{h}^{\circ}\right)$, com o auxílio de 
um colorímetro modelo CR 400 (Konica Minolta, Tóquio, Japão). A firmeza de polpa foi determinada em 2 superfícies opostas da região equatorial dos frutos, após a remoção de uma pequena porção da casca, com o auxílio de um penetrômetro eletrônico munido de ponteira de 7,9 mm de diâmetro (GÜSS Manufacturing Ltd., Cidade do Cabo, África do Sul). Os valores de firmeza de polpa foram expressos em $\mathrm{N}$. Os valores de AT foram determinados através de uma amostra de $10 \mathrm{~mL}$ de suco dos frutos, extraído de uma fatia longitudinal na porção central dos frutos, a qual foi diluída em $90 \mathrm{~mL}$ de água destilada e titulada com solução de $\mathrm{NaOH}$ 0,1 $\mathrm{N}$ até pH 8,1. Os resultados de AT foram expressos em \% de ácido málico. Os teores de SS ( ${ }^{\circ}$ Brix) foram determinados em um refratômetro digital, utilizando-se uma alíquota do suco obtido pelo processamento dos frutos.

Os frutos foram armazenados à temperatura de $-0,5 \pm 0,1^{\circ} \mathrm{C}$ e UR de $96 \pm 2 \%$, durante 9 meses em minicâmaras experimentais com capacidade de $233 \mathrm{~L}$. Foram utilizadas 5 condições de armazenamento, sendo elas: $0,5 \mathrm{kPa}$ de $\mathrm{O}_{2}+<0,03 \mathrm{kPa}$ de $\mathrm{CO}_{2} ; 1,0 \mathrm{kPa}$ de $\mathrm{O}_{2}+<0,03 \mathrm{kPa}$ de $\mathrm{CO}_{2}$; $1,0 \mathrm{kPa}$ de $\mathrm{O}_{2}+1,0 \mathrm{kPa}$ de $\mathrm{CO}_{2} ; 1,0 \mathrm{kPa}$ de $\mathrm{O}_{2}+2,0 \mathrm{kPa}$ de $\mathrm{CO}_{2}$ e $1,0 \mathrm{kPa}$ de $\mathrm{O}_{2}+3,0 \mathrm{kPa}$ de $\mathrm{CO}_{2}$.

As condições de AC foram estabelecidas mediante a diluição do $\mathrm{O}_{2}$ no ambiente de armazenamento com injeção de $\mathrm{N}_{2}$, proveniente de um gerador desse gás, que utiliza o princípio Pressure Swing Adsorption (PSA), e posterior injeção de $\mathrm{CO}_{2}$, proveniente de cilindros de alta pressão, até atingir as pressões parciais preestabelecidas nos tratamentos com $\mathrm{CO}_{2}>0,03 \mathrm{kPa}$. A manutenção das pressões parciais desejáveis dos gases, que variavam em razão da respiração dos frutos, foi realizada, diariamente, com o uso de equipamento automático para controle de gases (Kronenberger/Climasul, Caxias do Sul, Brasil). Quando as pressões parciais de $\mathrm{CO}_{2}$ e $\mathrm{O}_{2}$ não estavam adequadas, o equipamento procedia à correção até as pressões parciais preestabelecidas nos tratamentos. $\mathrm{O} \mathrm{O}_{2}$ consumido pela respiração foi reposto por meio da injeção de ar atmosférico nas minicâmaras, e o $\mathrm{CO}_{2}$ em excesso foi absorvido por uma solução de hidróxido de potássio a 40\%, através da qual foi circulado o ar do ambiente de armazenamento. Nos tratamentos com baixo $\mathrm{CO}_{2}(0,5 \mathrm{kPa}$ de $\mathrm{O}_{2}+<0,03 \mathrm{kPa}$ de $\mathrm{CO}_{2}$ e $1,0 \mathrm{kPa}$ de $\mathrm{O}_{2}+<0,03 \mathrm{kPa} \mathrm{CO}_{2}$ ), a pressão parcial de $\mathrm{CO}_{2}$ foi mantida por meio da colocação de cal hidratada no interior das minicâmaras, para a contínua eliminação do $\mathrm{CO}_{2}$ no ambiente de armazenamento.

Após a saída da câmara, os frutos permaneceram durante 6 dias em condição ambiente $\left(20 \pm 5^{\circ} \mathrm{C}\right.$ e UR de $\left.63 \pm 2 \%\right)$, sendo, em seguida, avaliados em relação à incidência e severidade de escurecimento da polpa. Para tanto, realizou-se um corte na secção transversal dos mesmos, onde se considerou frutos com incidência aqueles que apresentassem regiões internas da polpa com qualquer tipo de escurecimento com ou sem a formação de cavidades características da presença do distúrbio (Franck et al. 2007). As peras foram, ainda, avaliadas quanto à severidade, de acordo com a seguinte escala: 1 = sem incidência de escurecimento da polpa; 2 = severidade de escurecimento da polpa leve, com até $10 \%$ da polpa afetada; 3 = severidade de escurecimento da polpa moderada, com 11 a $30 \%$ da polpa afetada; e $4=$ severidade de escurecimento severa, com mais de $30 \%$ da polpa afetada. O valor de severidade foi calculado pelo somatório dos produtos do número de frutos pela sua respectiva nota da escala, dividido pelo número total de frutos da amostra.

Após as avaliações, os frutos de todas as unidades experimentais foram separados em 2 grupos: sem e com incidência de escurecimento da polpa. Dentre as 20 unidades experimentais que foram armazenadas em AC, apenas 16 foram utilizadas para a análise das concentrações minerais, por terem apresentado, simultaneamente, frutos com e sem escurecimento da polpa. Uma vez que poucas repetições apresentaram simultaneamente frutos com todos os níveis da escala de severidade do distúrbio, não foram realizadas comparações das concentrações minerais da polpa nos diferentes graus de severidade.

Para a análise mineral, foi utilizada apenas a polpa na região distal (pistilar) dos frutos, descartando-se a casca e o tecido da região carpelar central. Optou-se pela utilização apenas da região distal dos frutos, uma vez que esta apresenta as menores concentrações de $\mathrm{Ca}$ em maçãs, onde os sintomas de bitter pit normalmente ocorrem e, portanto, é a mais recomendada para a avaliação de elementos minerais associados ao risco de ocorrência do distúrbio (Miqueloto et al. 2011). O processamento foi realizado com o auxílio de um mixer Braun Multiquick MR40. As concentrações de Ca, Mg e $\mathrm{K}$ foram quantificadas ( $\mathrm{mg} \cdot \mathrm{kg}^{-1} \mathrm{de}$ massa fresca) segundo adaptação da metodologia descrita por Amarante et al. (2006). Após as determinações, foram ainda calculadas as seguintes relações entre os nutrientes: $\mathrm{K} / \mathrm{Ca}, \mathrm{Mg} / \mathrm{Ca}$ e N/Ca.

Para determinação das concentrações de Ca, $\mathrm{Mg}$ e K, foram pesados 5,0 g de polpa fresca em uma balança analítica, os quais foram depositados em cadinhos de porcelana, sendo então conduzidos a um forno tipo mufla durante $5 \mathrm{~h}$, sob uma temperatura de $630^{\circ} \mathrm{C}$. Após retirar as amostras da mufla, 
adicionou-se às mesmas $15 \mathrm{~mL}$ de $\mathrm{HCl}$ a $1,8 \mathrm{~N}$, formando o extrato original.

Para a determinação de $\mathrm{Ca}$, retirou-se uma alíquota de $5 \mathrm{~mL}$ do extrato original e adicionou-se $5 \mathrm{~mL}$ de lantânio no interior de um tubo falcon de $15 \mathrm{~mL}$. Para a quantificação de $\mathrm{Mg}$, retirou-se $2 \mathrm{~mL}$ do extrato original e adicionou-se $10 \mathrm{~mL}$ de água destilada. Desta solução diluída, pipetou-se $5 \mathrm{~mL}$ e adicionou-se $5 \mathrm{~mL}$ de lantânio. Já as concentrações de $\mathrm{K}$ foram determinadas por meio de uma alíquota de $1,0 \mathrm{~mL}$ do extrato original, a qual foi diluída em $20 \mathrm{~mL}$ de água destilada. Após as diluições, para os 3 minerais, as amostras foram lidas em espectrofotômetro de absorção atômica, modelo Analyst 100, marca PerkinElmer (Waltham, EUA).

Para determinação de N, $2 \mathrm{~g}$ de polpa fresca dos frutos foram colocados em uma mistura de ácido sulfúrico concentrada e água oxigenada 30 volumes e mantidos a $350{ }^{\circ} \mathrm{C}$. As concentrações de $\mathrm{N}$ foram determinadas pelo método semimicro Kjeldahl, conforme descrito por Tedesco et al. (1995).

Para comparação entre as condições de AC com relação à incidência e severidade de escurecimento da polpa, o delineamento experimental utilizado foi o inteiramente casualizado, com 4 repetições e unidade experimental constituída por 30 frutos. Os dados foram submetidos à análise de variância (ANOVA), sendo que os dados em porcentagem foram previamente transformados pela fórmula: arco seno $[(x+1) / 100]^{1 / 2}$. As médias foram comparadas pelo teste de Tukey $(\mathrm{p}<0,05)$. Para comparação entre os frutos com e sem escurecimento da polpa, o delineamento experimental foi o inteiramente casualizado, constituído por 16 repetições. Os dados das concentrações minerais, bem como das relações $\mathrm{K} / \mathrm{Ca}, \mathrm{Mg} / \mathrm{Ca}$ e N/Ca, foram submetidos à ANOVA $(\mathrm{p}<0,05)$. Para identificação dos principais elementos minerais capazes de discriminar frutos com e sem o distúrbio, os dados foram submetidos à análise canônica discriminante (ACD), segundo proposto por Amarante et al. (2006). A significância da diferença entre valores médios dos coeficientes canônicos homogeneizados $(\mathrm{CCH})$ de frutos sem e com escurecimento da polpa foi avaliada através da ANOVA $(p<0,05)$. O parâmetro adotado para avaliar o efeito de separação gerada pelos elementos minerais entre frutos sem e com escurecimento da polpa foi o coeficiente da taxa de discriminação paralela (TDP). O coeficiente da TDP é obtido por meio do produto entre valores dos coeficientes canônicos padronizados (CCP) e os coeficientes de correlação canônica $(r)$. Para o coeficiente da TDP, valores de elementos minerais positivos indicam efeito de separação entre frutos sem e com o distúrbio, sendo que aqueles com os maiores valores apresentam maior peso na separação entre esses 2 grupos de frutos. Em todos os procedimentos estatísticos, foi utilizado o programa SAS (SAS Institute, Cary, EUA).

\section{RESULTADOS E DISCUSSÃO}

A colheita dos frutos foi realizada no estádio de maturação recomendado para a cultivar 'Rocha', considerando a firmeza de polpa, que deve apresentar valores entre 55 e $65 \mathrm{~N}$ (Cavaco et al. 2009). Os frutos apresentavam firmeza de polpa de $56,5 \mathrm{~N}$; SS de $12,9^{\circ}$ Brix; AT de $0,14 \%$ de ácido málico e ${ }^{\circ}$ da casca de 99,3.

$\mathrm{O}$ armazenamento sob $1,0 \mathrm{kPa} \mathrm{O}_{2}+3,0 \mathrm{kPa} \mathrm{CO}_{2}$ induziu maior incidência de escurecimento da polpa em relação às condições de $0,5 \mathrm{kPa} \mathrm{O}_{2}+<0,03 \mathrm{kPa} \mathrm{CO}_{2}$ e $1,0 \mathrm{kPa} \mathrm{O}_{2}+$ $<0,03 \mathrm{kPa} \mathrm{CO}_{2}$, não diferindo, contudo, das demais condições avaliadas (Tabela 1). Com relação à severidade do distúrbio, os frutos armazenados na condição de $1,0 \mathrm{kPa}_{2}+3,0 \mathrm{kPa} \mathrm{CO}_{2}$ apresentaram maiores valores comparativamente aos frutos armazenados nas demais condições. As trocas gasosas influenciam no desenvolvimento do escurecimento da polpa em peras armazenadas em AC (Franck et al. 2007). Sob condições de alto $\mathrm{CO}_{2}$ na câmara, as pressões parciais desse gás podem atingir níveis ainda mais elevados nas células do interior da polpa, limitando a difusão do $\mathrm{O}_{2}$ para o interior do fruto (Pedreschi et al. 2008). Assim, a respiração anaeróbica pode ser induzida, levando à formação de compostos tóxicos relacionados ao metabolismo fermentativo $\mathrm{e}$, consequentemente, à ocorrência do escurecimento da

Tabela 1. Incidência e severidade de escurecimento da polpa em peras 'Rocha' submetidas a diferentes condições de armazenamento em atmosfera controlada, por 9 meses $\left(-0,5 \pm 0,1^{\circ} \mathrm{C}\right.$ e UR de $\left.96 \pm 2 \%\right)$, e mantidas durante 6 dias em condição ambiente $\left(20 \pm 5^{\circ} \mathrm{C}\right.$ e UR de $63 \pm 2 \%$ ).

\begin{tabular}{ccc}
\hline $\mathbf{O}_{\mathbf{2}}+\mathbf{C O}_{\mathbf{2}} \mathbf{( k P a )}$ & Incidência (\%) & Severidade (1-4) \\
\hline $0,5+<0,03$ & $6,20 \mathrm{~b}$ & $1,09 \mathrm{~b}$ \\
\hline $1,0+<0,03$ & $8,88 \mathrm{~b}$ & $1,12 \mathrm{~b}$ \\
\hline $1,0+1,0$ & $18,21 \mathrm{ab}$ & $1,32 \mathrm{~b}$ \\
\hline $1,0+2,0$ & $26,52 \mathrm{ab}$ & $1,39 \mathrm{~b}$ \\
\hline $1,0+3,0$ & $48,02 \mathrm{a}$ & $1,79 \mathrm{a}$ \\
\hline $\mathrm{CV}(\%)$ & 35,3 & 10,8 \\
\hline
\end{tabular}

Médias seguidas pela mesma letra na vertical não diferem entre si pelo teste de Tukey $(p<0,05) \cdot C V=$ Coeficiente de variação. 
polpa (Franck et al. 2007). Baixas pressões parciais de $\mathrm{O}_{2}$ nas células da polpa podem ainda ocasionar o acúmulo de espécies reativas de oxigênio, as quais, por sua vez, podem proporcionar a peroxidação lipídica e a desnaturação proteica (Deruchande et al. 2012), promovendo danos às membranas e, por conseguinte, induzindo a manifestação do distúrbio. Além disso, baixas pressões parciais de $\mathrm{O}_{2}$ e altas de $\mathrm{CO}_{2}$ causam redução do status energético na célula, com falta de ATP para restaurar as membranas, que, provavelmente, também pode ser fator determinante para induzir o escurecimento de polpa (Saquet et al. 2000; Veltman et al. 2003).

Menores concentrações de Ca foram observadas nos frutos que apresentaram escurecimento da polpa em relação àqueles que não apresentaram incidência do distúrbio (Tabela 2). Muitos distúrbios fisiológicos estão associados às baixas concentrações de Ca no fruto, tanto em peras (Raese e Drake 2006; Wójcik 2012) como em maçãs (James e Jobling 2009; Miqueloto et al. 2011). O Ca apresenta importante função na manutenção da integridade celular, uma vez que o mesmo estabelece ligações iônicas com o ânion fosfato de fosfolipídeos da membrana plasmática, contribuindo para estrutura e funcionalidade das membranas (Freitas et al. 2010; Amarante et al. 2013). Dessa forma, a deficiência de Ca pode apresentar grande relação com a ocorrência do escurecimento da polpa em peras europeias, já que a manifestação desse distúrbio é caracterizada pela redução da integridade de membranas e pelo processo de descompartimentalização celular (Saquet e Streif 2006; Franck et al. 2007).

Apesar de existirem poucos estudos que relacionem as concentrações minerais da polpa de peras com a incidência de distúrbios fisiológicos, alguns autores verificaram o efeito benéfico da aplicação pré-colheita de cloreto de cálcio na sua prevenção (Mahajan e Dhatt 2004; Raese e Drake 2006). Em um trabalho realizado com peras 'Conference', Wójcik (2012) observou que 6 aplicações pré-colheita de cloreto de cálcio proporcionaram frutos menos maduros e menos sensíveis ao escurecimento da polpa em relação àqueles que não foram submetidos ao tratamento. Segundo o autor, o aumento das concentrações de Ca na polpa retardou o processo de amadurecimento dos frutos, o que pode ter contribuído para uma redução na incidência do distúrbio, pois, de maneira geral, peras mais maduras são mais suscetíveis ao escurecimento da polpa (Franck et al. 2007).

Frutos com escurecimento da polpa apresentaram concentrações mais elevadas de K, bem como uma maior relação $\mathrm{K} / \mathrm{Ca}$, em comparação aos frutos sem escurecimento da polpa (Tabela 2). Alguns autores também observaram que altas concentrações de $\mathrm{K}$, bem como uma alta relação K/Ca na polpa, podem estar relacionadas com a ocorrência de escurecimento da polpa em maçãs 'Fuji' (Corrêa et al. 2012) e 'Braeburn' (Neuwald et al. 2008, 2014), após o armazenamento em AC. O K é um nutriente antagonista ao Ca, cujo efeito ocorre devido a uma competição por sítios de ligação na membrana plasmática sem, contudo, desempenhar a mesma função de manutenção da integridade de membranas que o $\mathrm{Ca}$, o que pode provocar o colapso de membranas e, consequentemente, a morte da célula (James e Jobling 2009; Freitas et al. 2010).

Para frutos com escurecimento da polpa, foram verificadas maiores relações $\mathrm{Mg} / \mathrm{Ca}$ e N/Ca em relação aos frutos que não tiveram incidência do distúrbio (Tabela 2). Possivelmente, essas maiores relações em frutos com o distúrbio se devem às baixas concentrações de $\mathrm{Ca}$, uma vez que as concentrações de $\mathrm{Mg}$ e de N, quando analisados

Tabela 2. Concentrações de Ca, $\mathrm{Mg}, \mathrm{K}$ e N ( $\mathrm{mg} \cdot \mathrm{kg}^{-1}$ de massa fresca) e valores das relações Mg/Ca, $\mathrm{K} / \mathrm{Ca}$ e N/Ca na polpa de peras 'Rocha' sem e com escurecimento da polpa.

\begin{tabular}{ccccc}
\hline Atributo mineral & Sem escurecimento & Com escurecimento & Probabilidade $^{*}$ & CV (\%) \\
\hline $\mathrm{Ca}$ & 96,20 & 84,27 & 0,030 & 16,4 \\
\hline $\mathrm{K}$ & $1.026,05$ & $1.105,74$ & 0,029 & 9,2 \\
\hline $\mathrm{Mg}$ & 78,93 & 77,00 & $\mathrm{~ns}$ & 12,1 \\
\hline $\mathrm{N}$ & 83,20 & 92,53 & $\mathrm{~ns}$ & 26,2 \\
\hline $\mathrm{K} / \mathrm{Ca}$ & 10,82 & 13,51 & $<0,001$ & 17,0 \\
\hline $\mathrm{Mg} / \mathrm{Ca}$ & 0,83 & 0,93 & 0,010 & 11,3 \\
\hline $\mathrm{N} / \mathrm{Ca}$ & 0,88 & 1,12 & 0,026 & 29,0 \\
\hline
\end{tabular}

*Probabilidade da diferença entre frutos sem e com escurecimento da polpa, para cada atributo mineral. CV = Coeficiente de variação; ns = Diferença não significativa $(p>0,05)$. 
individualmente, não diferiram entre frutos com e sem escurecimento da polpa (Tabela 2).

Como houve diferenças nas concentrações de $\mathrm{Ca}$ e $\mathrm{K}$, assim como nas relações $\mathrm{K} / \mathrm{Ca}, \mathrm{Mg} / \mathrm{Ca}$ e N/Ca, na análise univariada, entre frutos sem e com escurecimento da polpa, todos esses elementos foram submetidos à ACD.

O teste estatístico multivariado de Wilk's Lambda mostrou diferenças significativas entre frutos sem e com escurecimento da polpa na função canônica discriminante $1\left(\mathrm{FCD}_{1}\right)$ (Tabela 3). O valor da correlação canônica $(0,5927)$ indicou que existe associação entre os elementos minerais avaliados e a ocorrência desse distúrbio fisiológico em peras 'Rocha'.

A relação $\mathrm{K} / \mathrm{Ca}$ apresentou o maior valor de coeficiente da TDP (Tabela 3), indicando que, dentre todos os elementos minerais avaliados, esse é o que melhor discrimina peras 'Rocha' com e sem escurecimento da polpa. Por outro lado, a concentração isolada dos minerais $\mathrm{Ca}$ e $\mathrm{K}$ não se mostrou relevante na separação entre lotes de frutos com e sem a ocorrência do distúrbio, uma vez que valores negativos de coeficiente da TDP expressam efeito de supressão do atributo nutricional na separação entre frutos com e sem escurecimento da polpa na ACD (Amarante et al. 2006). Resultados semelhantes foram reportados por Miqueloto et al. (2011) em maçãs 'Fuji', os quais observaram que, apesar da diferença nas concentrações de Ca e K na polpa entre lotes com e sem a presença de bitter pit na análise univariada, esses minerais não seriam, isoladamente, os melhores parâmetros para promover uma distinção entre frutos com e sem o distúrbio, mas sim a relação K/Ca.

A representação gráfica entre os $\mathrm{CCP}$ da $\mathrm{FCD}_{1}$ mostra uma nítida separação entre frutos sem e com escurecimento da polpa (Figura 1). Frutos com escurecimento apresentaram

Tabela 3. Coeficientes da taxa de discriminação paralela para a função canônica discriminante $1\left(\mathrm{FCD}_{1}\right)$, referente aos atributos minerais da polpa de peras 'Rocha' sem e com escurecimento da polpa

\begin{tabular}{cc}
\hline Atributo mineral & $\begin{array}{c}\text { Taxa de discriminação } \\
\text { paralela }\end{array}$ \\
\hline $\mathrm{Ca}$ & $-0,4420$ \\
\hline $\mathrm{K}$ & $-0,0329$ \\
\hline $\mathrm{K} / \mathrm{Ca}$ & 1,4073 \\
\hline $\mathrm{Mg} / \mathrm{Ca}$ & $-0,0773$ \\
\hline $\mathrm{N} / \mathrm{Ca}$ & 0,1449 \\
\hline å & 1,0000 \\
\hline Correlação canônica & 0,5927 \\
\hline Probabilidade & 0,0004 \\
\hline
\end{tabular}

valores médios maiores de CCP em relação a frutos sem escurecimento da polpa (Figura 1), principalmente como resultado dos maiores valores da relação K/Ca na polpa.

Ao contrário do que acontece para os elementos $\mathrm{Mg}$, $\mathrm{K}$ e N, o Ca não é móvel no floema, sendo translocado na planta através do xilema (Miqueloto et al. 2014). Alguns autores relatam que, para algumas cultivares de maçã, ocorre drástica redução na concentração de Ca dos frutos em função da perda da funcionalidade do xilema (Dražeta et al. 2004; Miqueloto et al. 2014). Por outro lado, a funcionalidade do floema mantém-se inalterada, de forma que esse tecido continua a transportar $\mathrm{K}, \mathrm{Mg}$ e $\mathrm{N}$ durante todo o processo de desenvolvimento do fruto, garantindo elevados níveis desses nutrientes na polpa. Assim, nutrientes como Mg e K podem competir por sítios de ligação do Ca na membrana. Porém, tanto o Mg quanto o K não desempenham a mesma função na manutenção da integridade de membranas que o Ca, e o aumento nas concentrações de $\mathrm{Mg}$ e $\mathrm{K}$, relativo às concentrações de Ca na polpa, predispõem à ocorrência de bitter pit em maçãs (Freitas et al. 2010; Miqueloto et al. 2011). Mecanismo semelhante pode ocorrer em peras europeias com escurecimento da polpa, tendo em vista que esse distúrbio também está relacionado às menores

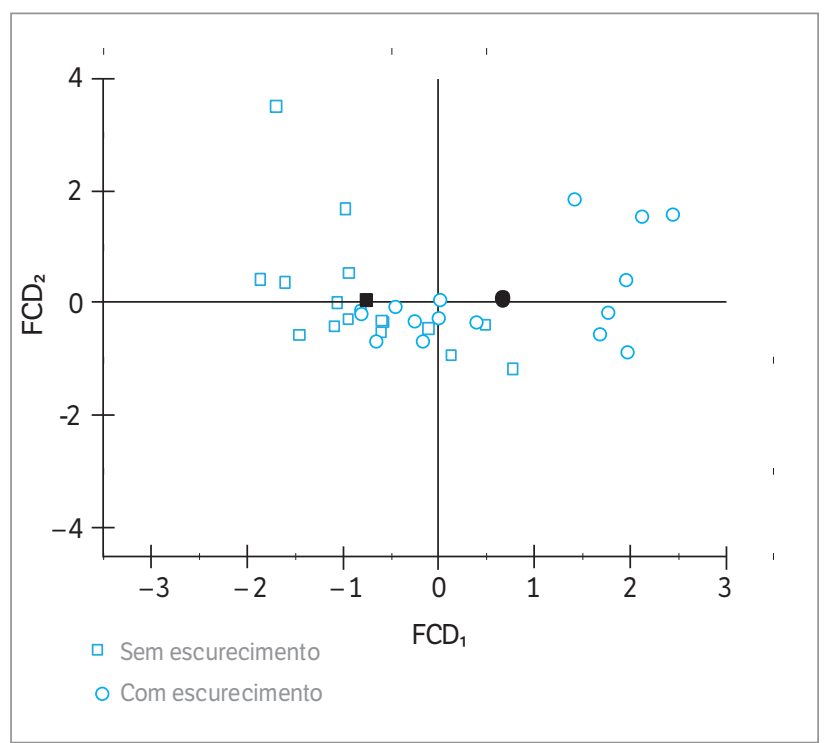

Figura 1. Coeficientes canônicos padronizados (CCP) da função canônica discriminante $1\left(\mathrm{FCD}_{1}\right)$, em peras 'Rocha' sem e com escurecimento da polpa, considerando os atributos minerais descritos na Tabela 3. Símbolo cheio representa o valor médio de CCP para frutos sem e com escurecimento da polpa. Uma vez que a análise permite apenas uma função canônica discriminante $\left(F D_{1}\right)$, uma segunda função canônica discriminante $\left(F C D_{2}\right)$ foi criada com observações aleatórias $[\mathrm{N}(0,1)]$, apenas para fins de representação gráfica. 
concentrações de Ca na polpa, como ocorre com o bitter pit em maçãs.

Os baixos níveis de Ca nos frutos, mesmo para solos devidamente adubados, também podem ser atribuídos à baixa capacidade de transporte desse nutriente para determinadas regiões da planta, uma vez que outros tecidos da pereira, que crescem com maior vigor, podem competir diretamente com os frutos pelo Ca (Saure 2005). Dentro de uma mesma planta, a posição do fruto na árvore pode influenciar a suscetibilidade do mesmo ao escurecimento da polpa (Franck et al. 2007). Frutos em condição de menor incidência de luz (interior do dossel) podem ter seu fluxo transpiratório reduzido e, consequentemente, menor aporte de $\mathrm{Ca}$ em relação a frutos que estão sob maior incidência luminosa (Miqueloto et al. 2011). De acordo com Franck et al. (2003), peras 'Conference', localizadas no topo da árvore, também são mais suscetíveis ao escurecimento da polpa em relação a frutos localizados nas porções mais baixas do dossel.

A combinação de diversos fatores pré-colheita irá influenciar um conjunto de atributos específicos do fruto, os quais, por sua vez, determinarão a suscetibilidade ao escurecimento da polpa (Franck et al. 2007). Dessa forma, diferenças nas concentrações minerais, bem como na composição química do fruto como um todo, podem explicar as variações encontradas quanto à suscetibilidade ao distúrbio em um mesmo pomar, como foi verificado para o presente estudo. Assim, técnicas de manejo do pomar, que visem melhorar o estado nutricional dos frutos, devem ser adotadas com a finalidade de prevenir o escurecimento da polpa em peras.
Uma vez que se torna difícil prever a ocorrência de escurecimento da polpa em peras, o conhecimento de que o aumento da relação K/Ca favorece a incidência do distúrbio pode contribuir para predizer frutos que apresentam maior risco de desenvolverem o distúrbio em pós-colheita. Além disso, essa informação pode auxiliar no planejamento das condições e do período de armazenamento mais adequados ao armazenamento, de forma a obter frutos de maior qualidade com uma menor incidência de distúrbios fisiológicos.

\section{CONCLUSÃO}

O armazenamento sob $1,0 \mathrm{kPa} \mathrm{O}_{2}+3,0 \mathrm{kPa} \mathrm{CO}$ aumenta a incidência e severidade de escurecimento da polpa em peras 'Rocha'. O escurecimento da polpa em peras 'Rocha' está associado a menores concentrações de $\mathrm{Ca}$, bem como a concentrações mais elevadas de $\mathrm{K}$ e a maiores relações $\mathrm{Mg} / \mathrm{Ca}, \mathrm{K} / \mathrm{Ca}$ e N/Ca nos frutos. A relação K/Ca na polpa é o atributo mineral que melhor discrimina peras 'Rocha' com e sem escurecimento da polpa.

\section{AGRADECIMENTOS}

Os autores agradecem ao Conselho Nacional de Desenvolvimento Científico e Tecnológico (CNPq) e à Fundação de Amparo à Pesquisa e Inovação do Estado de Santa Catarina (FAPESC) pelo apoio financeiro a este projeto.

\section{REFERÊNCIAS}

Amarante, C. V. T., Chaves, D. V. e Ernani, P. R. (2006). Análise multivariada de atributos nutricionais associados ao "bitter pit" em maçãs 'Gala'. Pesquisa Agropecuária Brasileira, 41, 841846. http://dx.doi.org/10.1590/S0100-204X2006000500017.

Amarante, C. V. T., Miqueloto, A., Freitas, S. T., Steffens, C. A., Silveira, J. P. G. e Corrêa, T. R. (2013). Fruit sampling methods to quantify calcium and magnesium contents to predict bitter pit development in 'Fuji' apple: a multivariate approach. Scientia Horticulturae, 157, 19-23. http://dx.doi.org/10.1016/j. scienta.2013.03.021.
Amarante, C. V. T., Steffens, C. A. e Ernani, P. R. (2010). Identificação pré-colheita do risco de ocorrência de "bitter pit” em maçãs ‘Gala' por meio de infiltração com magnésio e análise dos teores de cálcio e nitrogênio nos frutos. Revista Brasileira de Fruticultura, 32, 27-34. http://dx.doi.org/10.1590/ S0100-29452010005000015.

Cavaco, A. M., Pinto, P., Antunes, M. D., Silva, J. M. e Guerra, R. (2009). 'Rocha' pear firmness predicted by a Vis/NIR segmented model. Postharvest Biology and Technology, 51, 311-319. http://dx.doi.org/10.1016/j.postharvbio.2008.08.013. 
Corrêa, T. R., Steffens, C. A., Amarante, C. V. T., Tanaka, H., Stanger, M. C., Brackmann, A. e Ernani, P. R. (2012). Composição mineral, qualidade e degenerescência de polpa de maçãs 'Fuji' em diferentes porta-enxertos durante armazenamento em atmosfera controlada. Revista Brasileira de Fruticultura, $34,33-40$.

Deruchande, T., Fidalgo, F., Larrigaudière, C. e Almeida, D. P. F. (2012). Internal browning disorders during storage of 'Rocha' pear: effects of harvest maturity and $\mathrm{CO}_{2}$ partial pressure. Proceedings of the $X$ Simposio Nacional y VII Ibérico sobre Maduración y Postcosecha; Lleida, Spain.

Dražeta, L., Lang, A., Hall, A. J. e Volz, R. K. (2004). Causes and effects of changes in xylem functionality in apple fruit. Annals of Botany, 93, 275-282. http://dx.doi.org/10.1093/aob/mch040.

Franck, C., Baetens, M., Lammertyn, J., Scheerlinck, N. e Nicolaï, B. M. (2003). Ascorbic acid mapping to study core breakdown. Postharvest Biology and Technology, 30, 133-142. http://dx.doi.org/10.1016/S0925-5214(03)00108-X.

Franck, C., Lammertyn, J., Ho, Q. T., Verboven, P. e Nicolaï, B. M. (2007). Browning disorders in pear fruit. Postharvest Biology and Technology, 43, 1-13. http://dx.doi.org/10.1016/j. postharvbio.2006.08.008.

Freitas, S. T., Amarante, C. V. T., Labavitch, J. M. e Mitcham, E. J. (2010). Cellular approach to understand bitter pit development in apple fruit. Postharvest Biology and Technology, 57, 613. http://dx.doi.org/10.1016/j.postharvbio.2010.02.006.

Galvis-Sánchez, A. C., Fonseca, S. C., Morais, A. M. M. B. e Malcata, F. X. (2004). Sensorial and physicochemical quality responses of pears (cv Rocha) to long-term storage under controlled atmospheres. Journal of the Science of Food and Agriculture, 84, 1646-1656. http://dx.doi.org/10.1002/jsfa.1798.

James, H. J. e Jobling, J. J. (2009). Contrasting the structure and morphology of the radial and diffuse flesh browning disorders and $\mathrm{CO}_{2}$ injury of 'Cripps Pink' apples. Postharvest Biology and Technology, 53, 36-42. http://dx.doi.org/10.1016/j. postharvbio.2009.02.001.

Lammertyn, J., Scheerlinck, N., Jancsók, P., Verlinden, B. E. e Nicolaï, B. M. (2003). A respiration-diffusion model for Conference pears I: model development and validation. Postharvest Biology and Technology, 30, 29-42. http://dx.doi. org/10.1016/S0925-5214(03)00061-9.
Mahajan, B. V. C. e Dhatt, A. S. (2004). Studies on postharvest calcium chloride application on storage behaviour and quality of Asian pear during cold storage. Journal of Food, Agriculture and Environment, 2, 157-159.

Martin, M. S., Steffens, C. A., Amarante, C. V. T., Brackmann, A. e Linke Junior, W. (2015). Qualidade de peras 'Rocha' armazenadas em atmosfera controlada. Revista Brasileira de Fruticultura, 37, 73-82. http://dx.doi.org/10.1590/0100-2945-017/14.

Miqueloto, A., Amarante, C. V. T., Steffens, C. A., Santos, A., Miqueloto, T. e Silveira, J. P. G. (2011). Atributos fisiológicos, físico-químicos e minerais associados à ocorrência de "bitter pit” em maçãs. Pesquisa Agropecuária Brasileira, 46, 689696. http://dx.doi.org/10.1590/S0100-204X2011000700003.

Miqueloto, A., Amarante, C. V. T., Steffens, C. A., Santos, A. e Mitcham, E. (2014). Relationship between xylem functionality, calcium content and the incidence of bitter pit in apple fruit. Scientia Horticulturae, 165, 319-323. http://dx.doi.org/10.1016/j. scienta.2013.11.029.

Neuwald, D. A., Kittemann, D. e Streif, J. (2008). Possible prediction of physiological storage disorders in 'Braeburn' apples comparing fruit of different orchards. Acta Horticulturae, 796, 211-216. http://dx.doi.org/10.17660/ActaHortic.2008.796.28.

Neuwald, D. A., Sestari, I., Kitteman, D., Streif, J., Weber, A. e Brackmann, A. (2014). Can mineral analysis be used as a tool to predict 'Braeburn' Browning Disorders (BBD) in apple in commercial controlled atmosphere (CA) storage in Central Europe? Erwerbs-Obstbau, 56, 35-41. http://dx.doi.org/10.1007/ s10341-014-0202-x.

Pedreschi, R., Hertog, M., Robben, J., Noben, J. P. e Nicolai, B. (2008). Physiological implications of controlled atmosphere storage of 'Conference' pears (Pyrus communis L.): A proteomic approach. Postharvest Biology and Technology, 50, 110-116. http://dx.doi.org/10.1016/j.postharvbio.2008.04.004.

Raese, J. T. e Drake, S. R. (2006). Calcium foliar sprays for control of alfalfa greening, cork spot, and hard end in 'Anjou' pears. Journal of Plant Nutrition, 29, 543-552. http://dx.doi. org/10.1080/01904160500526683.

Saquet, A. A. e Streif, J. (2006). Fermentative metabolism in 'Conference' pears under various storage conditions. The Journal of Horticultural Science and Biotechnology, 81, 910914. http://dx.doi.org/10.1080/14620316.2006.11512158. 
Saquet, A. A., Streif, J. e Bangerth, F. (2000). Changes in ATP, ADP and pyridine nucleotide levels related to the incidence of physiological disorders in 'Conference' pears and 'Jonagold' apples during controlled atmospherestorage. Journal of Horticultural Science and Biotechnology, 75, 243-249. http://dx.doi.org/10.1080/14620316.2000.11511231.

Saure, M. C. (2005). Calcium translocation to fleshy fruit: its mechanism and endogenous control. Scientia Horticulturae, 105, 65-89. http://dx.doi.org/10.1016/j.scienta.2004.10.003.

Tedesco, M. J., Gianello, C., Bissani, C. A., Bohnen, H. e Volkweiss, S. J. (1995). Análise do solo, planta e outros materiais. Porto Alegre: UFRGS.

Veltman, R. H., Lenthéric, I., van der Plas, L. H. W. e Peppelenbos, H. W. (2003). Internal browning in pear fruit (Pyrus communis
L. cv 'Conference') may be a result of a limited availability of energy and antioxidants. Postharvest Biology and Technology, 28, 295-302. http://dx.doi.org/10.1016/S0925-5214(02)00198-9. Wójcik, P. (2012). Quality and 'Conference' pear storability as influenced by preharvest sprays of calcium chloride. Journal of Plant Nutrition, 35, 1970-1983. http://dx.doi.org/10.1080/0 1904167.2012.716890.

Yan, S., Li, L., He, L., Liang, L. e Li, X. (2013). Maturity and cooling rate affects browning, polyphenol oxidase activity and gene expression of 'Yali' pears during storage. Postharvest Biology and Technology, 85, 39-44. http://dx.doi.org/10.1016/j.postharvbio.2013.04.016. 